\begin{tabular}{|c|c|}
\hline Title: & Theoretical Analysis of Synchronous Machines with Displaced Reluctance Axis \\
\hline Authors: & Patrick Winzer, Martin Doppelbauer \\
\hline Institute: & $\begin{array}{l}\text { Karlsruhe Institute of Technology (KIT) } \\
\text { Elektrotechnisches Institut (ETI) } \\
\text { Hybrid Electric Vehicles (HEV) }\end{array}$ \\
\hline Type: & Conference Proceedings \\
\hline Published at: & $\begin{array}{l}\text { Proceedings } 2014 \text { International Conference on Electrical Machines (ICEM), Berlin, } \\
\text { Germany, September 2-5, } 2014 \\
\text { Publisher: IEEE, Piscataway (NJ) } \\
\text { Year: } 2014 \\
\text { ISBN: } 978-1-4799-4389-0 \\
\text { Pages: } 641-647\end{array}$ \\
\hline Hyperlinks: & DOI: http://dx.doi.org/10.1109/ICELMACH.2014.6960248 \\
\hline
\end{tabular}

(C) 2014 IEEE. Personal use of this material is permitted. Permission from IEEE must be obtained for all other uses, in any current or future media, including reprinting/republishing this material for advertising or promotional purposes, creating new collective works, for resale or redistribution to servers or lists, or reuse of any copyrighted component of this work in other works. 


\title{
Theoretical Analysis of Synchronous Machines with Displaced Reluctance Axis
}

\author{
Patrick Winzer, Martin Doppelbauer
}

\begin{abstract}
This paper examines synchronous machines, whose excitation axis and reluctance axis can have an arbitrary angle. Basic equations for this machine type are set up and control strategies for maximum torque are derived. By using a normalized representation, it will be shown that machines with displacement angles of about $60^{\circ}$ need up to $10 \%$ less permanent magnet material than conventional machines while yielding comparable motor performance.
\end{abstract}

Index Terms -- Field weakening, synchronous machine, permanent magnet machines, variable speed drive, two-part rotor, displacement, electric traction, motor design

\section{INTRODUCTION}

$\mathrm{F}$ OR many applications, such as electric traction, good field weakening capabilities and a wide constant power speed range are required [1]. Thus, permanent magnet synchronous machines (PMSM) are usually equipped with interior permanent magnets (PM) [2]. Due to their magnetic properties, those machines provide the highest inductance electrically perpendicular to the direction of the rotor excitation, i.e. $L_{\mathrm{q}}>L_{\mathrm{d}}$. On the one hand, this fact can be used to increase machine performance by utilizing the reluctance torque and to reduce the use of PM material [2]. On the other hand, a low direct inductance $L_{\mathrm{d}}$ means that a high value of current is needed for flux weakening, which deteriorates the flux weakening performance of those machines [1].

To overcome this disadvantage, several authors have investigated different machine designs which provide $L_{\mathrm{q}}<L_{\mathrm{d}}$. In [3], Bianchi and Bolognani proposed a machine geometry with a segmented rotor structure and demonstrated its improved field weakening performance. In [1], Gosden et al. proposed a two-part rotor consisting of a reluctance part and a surface PM part, which are mounted axially on a common shaft in a way that the high inductance axis of the reluctance part and the excitation axis of the PM part are aligned to achieve $L_{\mathrm{q}}<L_{\mathrm{d}}$. However, to maintain performance of this class of PMSM, the relative PM flux linkage has to be increased by about $50 \%$ [3]. This implies increased cost and tightened safety issues due to the higher induced voltage.

Generally, the two rotor parts of a two-part rotor can be mounted at any chosen angle. Fig. 1 shows a simplified drawing of such a machine, consisting of a reluctance-free excitation part, which is described in the $\mathrm{d} / \mathrm{q}$ reference frame, and a reluctance part, which is described in the $\mathrm{r} / \mathrm{s}$ reference frame and contains the resulting reluctance of both rotor parts. These two systems are displaced by the

P. Winzer is with Karlsruhe Institute of Technology, Karlsruhe, Germany (e-mail: patrick.winzer@kit.edu).

M. Doppelbauer is with Karlsruhe Institute of Technology, Karlsruhe, Germany (e-mail: martin.doppelbauer@kit.edu).

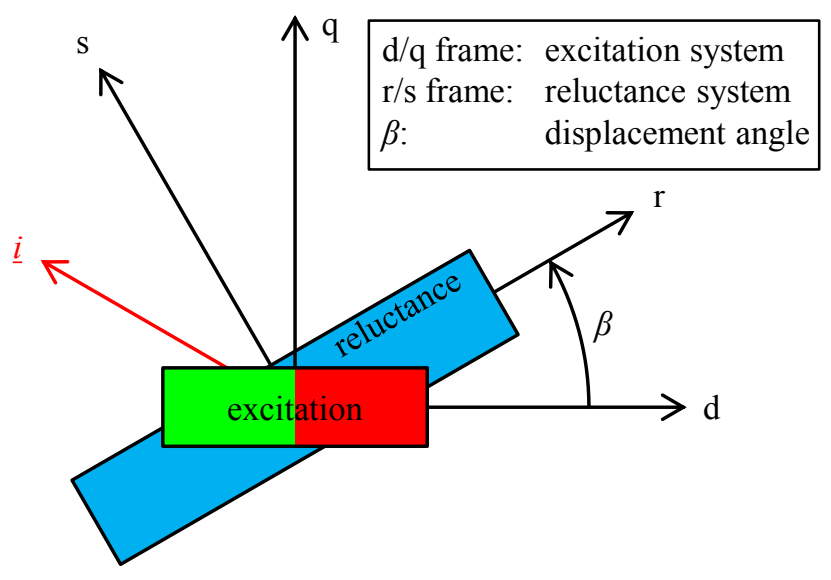

Fig. 1. Basic reference frames of machines with displaced reluctance axis

angle $\beta$. Using this representation, conventional PM synchronous machines with $L_{\mathrm{q}}>L_{\mathrm{d}}$ have $\beta=90^{\circ}$, machines with $L_{\mathrm{q}}<L_{\mathrm{d}}$ have $\beta=0^{\circ}$.

The two-part rotor was investigated in more detail in [4] by Chalmers et al., wherein a set of base equations was established to describe this motor dependent on the displacement angle $\beta$ between the two rotor parts. Unfortunately, the authors of [1] and [4] discarded the possibility to set any desired displacement angle in their following contributions [5], [6], [7] partly due to the fact that these machines don't show the same characteristics in motoring and generating mode.

Nevertheless, subsequent work has been done by other authors on this topic. A theoretical study was carried out by Randi and Astier [8], who used the displacement angle to synthesize a machine with desired characteristics consisting of two different rotor parts, however ending up with a conventional PMSM exhibiting $L_{\mathrm{q}}>L_{\mathrm{d}}$. Practical work has been accomplished by Chen [9] and Beser et al. [10]. Chen analyzed displacement angles of $\beta= \pm 20^{\circ}$ and identified different field weakening behavior, Beser studied an even broader range of displacement angles, yet both are lacking of a theory describing the phenomena they discovered.

This paper analyzes the potential of machines with displaced reluctance axis. It is motivated by applications which mostly require just one operation mode, i.e. motoring or generating, such as traction drives. Assuming that any desired displacement angle can be realized, this paper first sets up a basic set of equations in Chapter II. Following that, machine characteristics are illustrated in Chapter III and operating strategies are developed in Chapter IV. From a set of possible motor configurations, design planes are derived and evaluated to find the optimal parameter set for motors in Chapter V. 


\section{BASIC MACHINE EQUATIONS}

\section{A. Assumptions}

This paper aims at investigating the fundamental behavior of synchronous machines with displaced reluctance axis. In order to keep the system as simple as possible, a few assumptions have to be made [7]:

- All losses are zero, especially the stator resistance is set to zero;

- The iron and PMs are considered linear (i.e. saturation is neglected), so that linear inductances can be defined;

- Harmonics and other parasitic effects are not taken into account, electrical quantities are sinusoidal and there is no torque ripple.

\section{B. Normalization}

The equation system will be derived in normalized form according to Soong and Miller [2]. As opposed to [7], base quantities are rated current $I_{\mathrm{B}}$, rated voltage $V_{\mathrm{B}}$ (both RMS phase quantities) and rated motoring speed $\omega_{B}$, at which both $I_{\mathrm{B}}$ and $V_{\mathrm{B}}$ appear while the motor is operated applying the maximum torque per ampere (MTPA, [4]) strategy.

This set of base quantities is advantageous when comparing rotor concepts while the drive system including the stator remains the same. The inverter specifies the values $I_{\mathrm{B}}$ and $V_{\mathrm{B}}$, the application sets $\omega_{\mathrm{B}}$. This means constant base flux linkage $\Psi_{\mathrm{B}}=V_{\mathrm{B}} / \omega_{\mathrm{B}}$, constant base inductance $L_{\mathrm{B}}=\Psi_{\mathrm{B}} / I_{\mathrm{B}}$, constant base torque $T_{\mathrm{B}}=$ $\frac{m}{2} p \Psi_{\mathrm{B}} I_{\mathrm{B}}$ and constant base power $P_{\mathrm{B}}=\frac{m}{2} V_{\mathrm{B}} I_{\mathrm{B}}$ with the number of pole pairs $p$ and the number of phases $m$.

The motor is assumed to be fed by a perfect $m$-phase current source so that any current vector can be applied. Stator voltage is then assumed as a resulting quantity.

\section{Normalized Equation System}

Any chosen space vector, e.g. the normalized current vector $\underline{i}$, can be represented in the $\mathrm{d} / \mathrm{q}$ reference frame or the r/s reference frame by its components (see Fig. 1). d/q components can be expressed in the $\mathrm{r} / \mathrm{s}$ reference frame via

$$
\begin{gathered}
i_{\mathrm{r}}=i_{\mathrm{d}} \cos \beta+i_{\mathrm{q}} \sin \beta, \\
i_{\mathrm{s}}=-i_{\mathrm{d}} \sin \beta+i_{\mathrm{q}} \cos \beta
\end{gathered}
$$

where the absolute value of the vector equals

$$
i=\sqrt{i_{\mathrm{r}}^{2}+i_{\mathrm{s}}^{2}} .
$$

Defining the saliency

$$
\zeta=l_{\mathrm{r}} / l_{\mathrm{s}}
$$

from the normalized inductances $l_{\mathrm{r}}$ and $l_{\mathrm{s}}$ that appear in the $\mathrm{r} / \mathrm{s}$ reference frame, normalized stator flux linkage components $\psi_{\mathrm{r}}$ and $\psi_{\mathrm{s}}$ can be written as

$$
\begin{gathered}
\psi_{\mathrm{r}}=\psi_{\mathrm{PM}} \cos \beta+l_{\mathrm{r}} i_{\mathrm{r}} \\
\psi_{\mathrm{s}}=-\psi_{\mathrm{PM}} \sin \beta+l_{\mathrm{r}} \zeta^{-1} i_{\mathrm{s}},
\end{gathered}
$$

where $\psi_{\mathrm{PM}}$ is transformed into the $\mathrm{r} / \mathrm{s}$ reference frame using (1) and (2). Regarding Section II $A$ and introducing the normalized speed $\omega$, normalized voltage equations are

$$
v_{\mathrm{r}}=-\omega \psi_{\mathrm{s}}=-\omega\left(-\psi_{\mathrm{PM}} \sin \beta+l_{\mathrm{r}} \zeta^{-1} i_{\mathrm{s}}\right),
$$

$$
v_{\mathrm{s}}=\omega \psi_{\mathrm{r}}=\omega\left(\psi_{\mathrm{PM}} \cos \beta+l_{\mathrm{r}} i_{\mathrm{r}}\right)
$$

Similar to (3), the absolute value of the voltage vector is

$$
v=\sqrt{v_{\mathrm{r}}^{2}+v_{\mathrm{s}}^{2}}
$$

Normalized torque $t$ can be derived from the general torque equation $t=\psi^{*} \underline{i}=\psi_{\mathrm{r}} i_{\mathrm{s}}-\psi_{\mathrm{s}} i_{\mathrm{r}}$ using

$$
\zeta^{\prime}=1-\zeta^{-1}
$$

which yields

$$
t=\psi_{\mathrm{PM}}\left(i_{\mathrm{s}} \cos \beta+i_{\mathrm{r}} \sin \beta\right)+l_{\mathrm{r}} \zeta^{\prime} i_{\mathrm{r}} i_{\mathrm{s}} .
$$

The first summand of (11) represents the torque $t_{\mathrm{PM}}=\psi_{\mathrm{PM}} i_{\mathrm{d}}$ which is created by the PM, the second summand is due to the salient nature of the reluctance part.

Normalized power is expressed by

$$
p=t \omega=v_{\mathrm{r}} i_{\mathrm{r}}+v_{\mathrm{s}} i_{\mathrm{s}}
$$

for both the electrical and the mechanical part due to the assumed absence of losses.

Setting up the basic equation system in the $d / q$ reference frame turned out to be unfavorable as equations are more complex and inductances in the $\mathrm{d} / \mathrm{q}$ reference frame are a function of the current.

\section{Calculation of $l_{r}$}

Equations (5)-(12) describe the basic machine behavior. They are dependent on four machine parameters: the normalized PM flux linkage $\psi_{\mathrm{PM}}$, the saliency $\zeta$, the displacement angle $\beta$ and the normalized inductance $l_{\mathrm{r}}$.

In the case of $\beta=0^{\circ}$ and $\beta=90^{\circ}$, an analytic equation can be found to express $l_{\mathrm{r}}$ as a function of $\psi_{\mathrm{PM}}$ and $\zeta$ [11]. Therefore, there are only two independent parameters that describe a machine entirely.

In the case of arbitraty $\beta, l_{\mathrm{r}}$ can also be eliminated, so that the three parameters $\psi_{\mathrm{PM}}, \zeta$ and $\beta$ are sufficient. However, in this case, an analytical equation for $l_{\mathrm{r}}$ does not exist. Thus, the problem has to be solved numerically by finding a solution for $l_{\mathrm{r}}$ which yields $\sqrt{\psi_{\mathrm{r}}^{2}+\psi_{\mathrm{s}}^{2}}=1 \mathrm{using}$ (5) and (6) while applying MTPA strategy (see Section IV $B)$. This can be achieved using constrained nonlinear numerical optimization techniques.

\section{E. Machine Type Depending on $\beta$}

Analyzing the minima and maxima of the torque versus the current angle $\gamma_{\mathrm{rs}}=$ atan $\mathrm{i}_{\mathrm{s}} / i_{\mathrm{r}}$ numerically at maximum current $i=1$ using (11) and (3) and assuming $\psi_{\mathrm{PM}}>0$, $\zeta>1$ and $\omega \geq 0$, some substantial statements can be made:

- Machines with $\beta=0^{\circ}$ or $\beta=90^{\circ}$ show equal absolute maximum torque in both motoring and generating mode;

- Machines with $0^{\circ}<\beta<90^{\circ}$ exhibit greater absolute maximum torque in motoring mode;

- Machines with $-90^{\circ}<\beta<0^{\circ}$ and $90^{\circ}<\beta<$ $180^{\circ}$ exhibit greater absolute maximum torque in generating mode.

Due to the electromagnetic and mechanical symmetry of the reluctance part, $\beta$ can be limited to a range of $180^{\circ}$. This paper uses $-45^{\circ} \leq \beta<135^{\circ}$. 


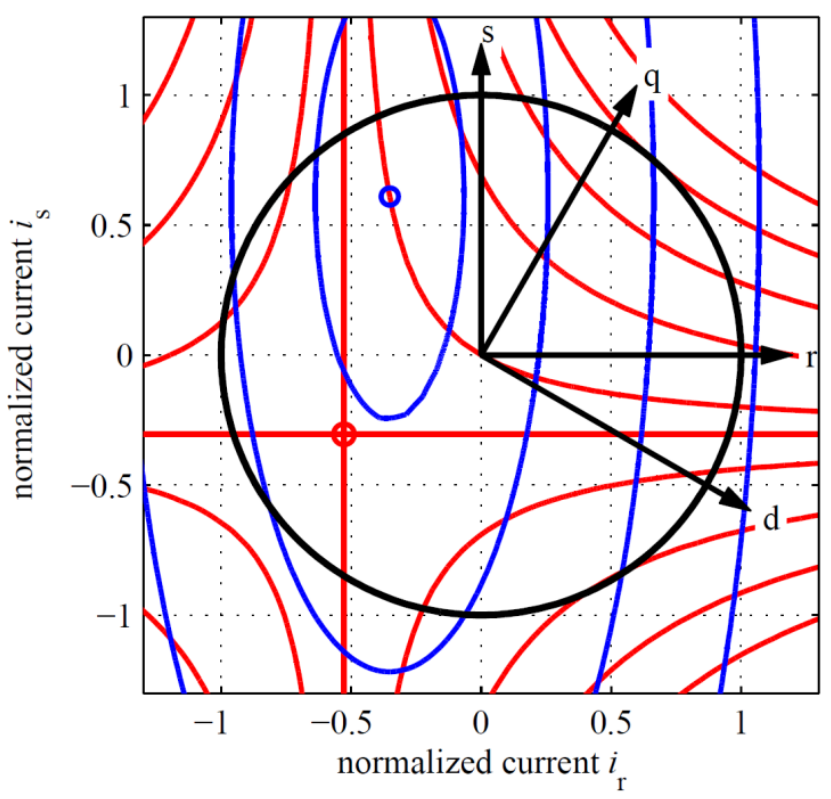

Fig. 2. Circle diagram of a machine with $\psi_{\mathrm{PM}}=0.4, \zeta=3, \beta=30^{\circ}$. Black: maximum current circle. Red: hyperbolas of constant torque. Blue: ellipses of constant voltage

\section{THE CIRCLE DiAgRAM}

The content of the Chapters III and IV will be discussed on an example machine with $\psi_{\mathrm{PM}}=0.4, \zeta=3$ and $\beta=30^{\circ}$. Fig. 2 shows the circle diagram of this machine.

The black current circle surrounds the area where $i \leq 1$, which represents the allowed area of operation.

The red hyperbolas indicate levels of constant torque according to (11). The function is point symmetric to its saddle point, which is marked with a red circle. The torque hyperbolas have a vertical and a horizontal asymptote, which pass the saddle point. The saddle point of conventional PMSM lies on the direct axis and yields $t=0$. In general, the torque on the saddle point is different from zero. It is the hyperbola passing $i=0$ that yields $t=0$.

The blue ellipses in Fig. 2 represent points of constant voltage for a fixed speed [12]. Any point inside an ellipsis is feasible for the corresponding speed. Increasing the speed leads to contracting ellipses. As opposed to conventional PMSM, their center $\left(i_{\mathrm{r} \infty}, i_{\mathrm{s} \infty}\right)$, which is indicated by a blue circle, does not necessarily lie on an axis as well. It is calculated by plugging $v_{\mathrm{r}}=v_{\mathrm{s}}=0$ in (7) and (8):

$$
\begin{gathered}
i_{\mathrm{r} \infty}=-\frac{\psi_{\mathrm{PM}}}{l_{\mathrm{r}}} \cos \beta, \\
i_{\mathrm{s} \infty}=\frac{\psi_{\mathrm{PM}}}{l_{\mathrm{r}}} \zeta \sin \beta, \\
i_{\infty}=\sqrt{i_{\mathrm{r} \infty}^{2}+i_{\mathrm{s} \infty}^{2}}=\frac{\psi_{\mathrm{PM}}}{l_{\mathrm{r}}} \sqrt{\cos ^{2} \beta+\zeta^{2} \sin ^{2} \beta} .
\end{gathered}
$$

The center of the ellipses lies on the torque trajectory which passes $i=0$, because torque at infinite speed must be zero due to the limitation of $p \leq 1$. Yet, as opposed to conventional PMSM, reluctance torque and PM torque itself are not zero at that point in general, which becomes clear when substituting (13)-(14) into (11). Rather, both summands yield exactly opposing torque.

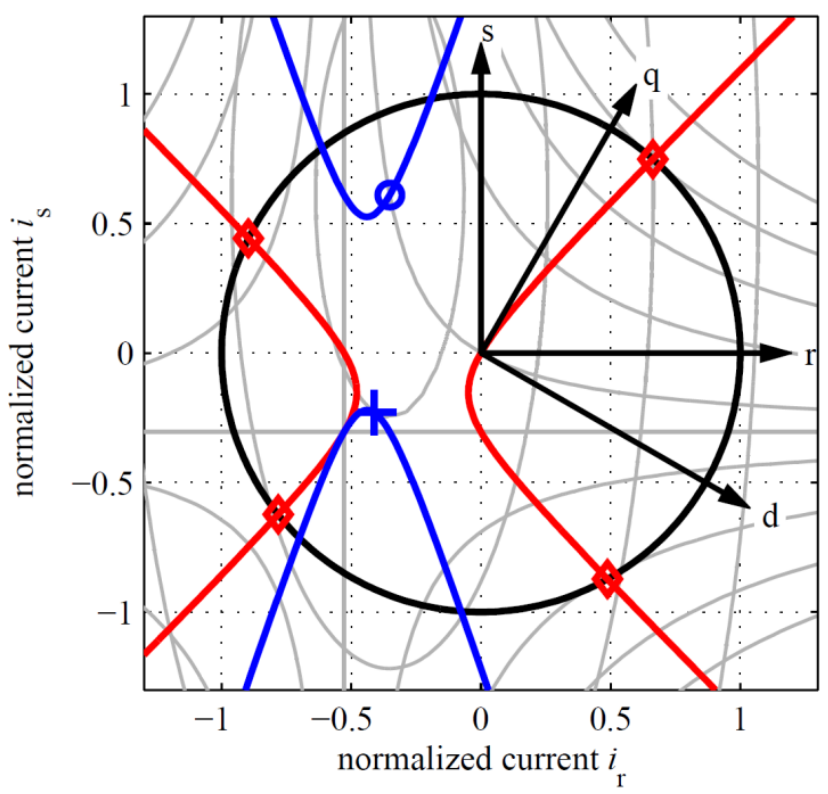

Fig. 3. Trajectories of MTPA (red) and MTPV (blue) strategy. Blue Circle: point $\left(i_{\mathrm{r} \infty}, i_{\mathrm{s} \infty}\right)$. Blue plus sign: point $\left(i_{\mathrm{r}+}, i_{\mathrm{s}+}\right)$ (see Section IV $D$ ). Diamonds: MTPA points (see Section IV $B$ ).

\section{OPERATING STRATEGIES}

This Chapter aims at deriving a strategy to achieve the maximum absolute torque for motoring and generating mode at any possible speed. The required currents change with speed due to contracting voltage ellipses and create trajectories in the $i_{\mathrm{r}}-i_{\mathrm{s}}$ - plane. In the following, these will be referenced as maximum torque trajectories.

As mentioned in Section II $E$, machines with arbitrary $\beta$ exhibit different torque capabilities in motoring and generating mode and hence have different maximum torque trajectories. Thus, both modes have to be analyzed separately.

Furthermore, as will become clear in Section IV $G$, it is necessary to analyze up to two further maximum torque trajectories that can be neglected for conventional PMSM.

\section{A. Modes of Operation}

In order to calculate the different maximum torque trajectories, different operation modes have to be analyzed. As for conventional PMSM [4], there are three operation modes:

- Mode 1 operation: current is limited while the voltage is lower than its maximum value, i.e. $i=1$ and $v<1$.

- Mode 2 operation: current and voltage are limited, i.e. $i=1$ and $v=1$.

- Mode 3 operation: only voltage is limited, i.e. $i<1$ and $v=1$.

\section{B. $\quad$ Mode 1 Operation (Maximum Torque per Ampere)}

For any current $i$ there are several combinations of $i_{\mathrm{r}}$ and $i_{\mathrm{s}}$ yielding local maximum or minimum torque. Together, these maxima and minima form the MTPA trajectories, which are plotted red in Fig. 3. They are calculated by using analytical optimization by means of 
Lagrange multipliers. Therefore, gradients of an objective function and an equality constraint function have to be calculated and compared. When they are parallel, a feasible point has been found [12].

Equation (11) serves as objective function, (3) is the constraint function. Both are differentiated with respect to $i_{\mathrm{r}}$ and $i_{\mathrm{s}}$ and compared, yielding the equation for the MTPA trajectories:

$$
\frac{\psi_{\mathrm{PM}} \sin \beta+l_{\mathrm{r}} \zeta^{\prime} i_{\mathrm{s}}}{\psi_{\mathrm{PM}} \cos \beta+l_{\mathrm{r}} \zeta^{\prime} i_{\mathrm{r}}}=\frac{i_{\mathrm{r}}}{i_{\mathrm{s}}}
$$

This equation can be solved for either $i_{\mathrm{r}}$ or $i_{\mathrm{s}}$, yielding two equations due to its quadratic nature. As can be seen from Figs. 2 and 3, one trajectory passes the origin (hereafter called primary MTPA trajectory), another trajectory passes the saddle point of the torque hyperbolas (hereafter called secondary MTPA trajectory). The primary MTPA trajectory represents global maximum and minimum torque, the secondary MTPA trajectory represents local maximum and minimum torque for a fixed value of $i$.

To find the intersection points of the MTPA trajectories with the current circle, (3) and (16) are solved with respect to $i_{\mathrm{r}}$ and $i_{\mathrm{s}}$ and with $i=1$. This yields a quartic function, which can be solved either analytically or numerically. Its solutions are the MTPA points, which are marked with diamond symbols in Fig. 3. If the secondary MTPA trajectory runs partly through the current circle, there are four solutions. The two corresponding MTPA points are called the secondary MTPA points.

The rated operating point is where the primary MTPA trajectory intersects the current circle, evoking positive torque. It can be maintained until speed increases rated speed, i.e. $\omega=1$, as this is the base speed of the normalization. In general, the other MTPA points yield different maximum speeds. Their equation can be derived from (7)-(9):

$$
\omega=\frac{v}{\sqrt{\left(-\psi_{\mathrm{PM}} \sin \beta+\frac{l_{\mathrm{r}}}{\zeta} i_{\mathrm{s}}\right)^{2}+\left(\psi_{\mathrm{PM}} \cos \beta+l_{\mathrm{r}} i_{\mathrm{r}}\right)^{2}}}
$$

where $v=1$.

\section{Mode 2 Operation}

After the maximum speed of an MTPA point has been exceeded, mode 2 operation commences. As the current magnitude is still 1 , the trajectory of mode 2 operation is equal to the current circle (see Fig. 3, black circle).

To find the current for a specific speed $\omega$, (1)-(3) and (7)-(9) with $v=1$ and $i=1$ have to be solved. This also yields a quartic function, providing up to four solutions. The correct value that belongs to the desired maximum torque trajectory has to be found by case analysis. The solution represents the intersection of the current circle with the speed-dependent voltage ellipsis.

\section{Mode 3 Operation (Maximum Torque per Voltage)}

Maximum torque per voltage trajectories (MTPV, [4]) are calculated in a similar manner to the MTPA trajectories. The objective function remains the same; the constraint function is given by (9), incorporating (7) and (8). This yields the equation for the MTPV trajectories:

$$
\frac{\psi_{\mathrm{PM}} \sin \beta+l_{\mathrm{r}} \zeta^{\prime} i_{\mathrm{s}}}{\psi_{\mathrm{PM}} \cos \beta+l_{\mathrm{r}} \zeta^{\prime} i_{\mathrm{r}}}=\zeta^{2} \frac{\psi_{\mathrm{PM}} l_{\mathrm{r}}^{-1} \cos \beta+i_{\mathrm{r}}}{-\psi_{\mathrm{PM}} \zeta_{\mathrm{r}}^{-1} \sin \beta+i_{\mathrm{s}}}
$$

Again, solving for $i_{\mathrm{r}}$ respectively $i_{\mathrm{s}}$ yields two equations, plotted as blue lines in Fig. 3. The hereafter called primary MTPV trajectory passes the point $\left(i_{\mathrm{r} \infty}, i_{\mathrm{s} \infty}\right)$ (see (13)-(14)), which is marked with a blue circle. The secondary MTPV trajectory passes the saddle point of the torque hyperbolas as well as the secondary MTPA trajectory. This trajectory also contains a boundary point $\left(i_{\mathrm{r}+}, i_{\mathrm{s}+}\right)$ with the voltage ellipses, marked with a plus sign, where the trajectory and a voltage ellipsis only touch in one point. Increasing speed means that there is no longer a feasible point on the secondary MTPV trajectory, signifying that there is a maximum speed for maximum torque trajectories containing this segment of the secondary MTPV trajectory. The relevance of this point is clarified in Section IV $G$.

To calculate the MTPV operating point of a specific speed $\omega,(7)-(9)$ and (18) have to be solved for $i_{\mathrm{r}}$ and $i_{\mathrm{s}}$, yielding a quartic function as well. Again, the desired value has to be found by case differentiation. The solution represents the intersection of the MTPV trajectories with the speed-dependent voltage ellipsis.

\section{E. Maximum Speed}

As with conventional PMSM, two types of machines can be distinguished: those theoretically capable of infinite speed and those with a specific maximum speed [2]. If $i_{\infty} \leq 1$, (see (15)), as is the case in the introduced example machine, the former is the case. If $i_{\infty}>1$, the machine does have a maximum speed. At that speed, the current circle and the corresponding voltage ellipsis have one common boundary point. It can also be calculated by analytical optimization, comparing the gradients of (3) and (7)-(9). This yields

$$
\zeta^{2} \frac{\psi_{\mathrm{PM}} l_{\mathrm{r}}^{-1} \cos \beta+i_{\mathrm{r}}}{-\psi_{\mathrm{PM}} \zeta l_{\mathrm{r}}^{-1} \sin \beta+i_{\mathrm{s}}}=\frac{i_{\mathrm{r}}}{i_{\mathrm{s}}} .
$$

Solving (3) and (19) with $i=1$ with respect to $i_{\mathrm{r}}$ and $i_{\mathrm{s}}$ yields a quartic function. Its solutions are the boundary points of the voltage ellipses with the current circle. To find the maximum speed, the solutions have to be plugged in (17). The highest speed is the maximum speed, as lower speeds mean that there must be an intersection of the current circle ant the corresponding voltage ellipsis, signifying that there are several feasible operation points. As opposed to conventional PMSM, the torque at maximum speed is not necessarily zero.

\section{F. $\quad$ Mode Order}

When raising speed, the mode order of the maximum torque trajectories of conventional PMSM is mode 1 mode 2 when $i_{\infty} \geq 1$ respectively mode 1 - mode 2 - mode 3 when $i_{\infty}<1$ [2]. However, this strict rule does not necessarily apply to machines with an arbitrary displacement angle $\beta$. It is possible that mode 2 follows mode 3 again once or twice, even if the machine has a finite maximum speed. This is due to the shifted positions of the MTPV trajectories. In that case, when entering mode 3, an algorithm has to check if the corresponding MTPV 


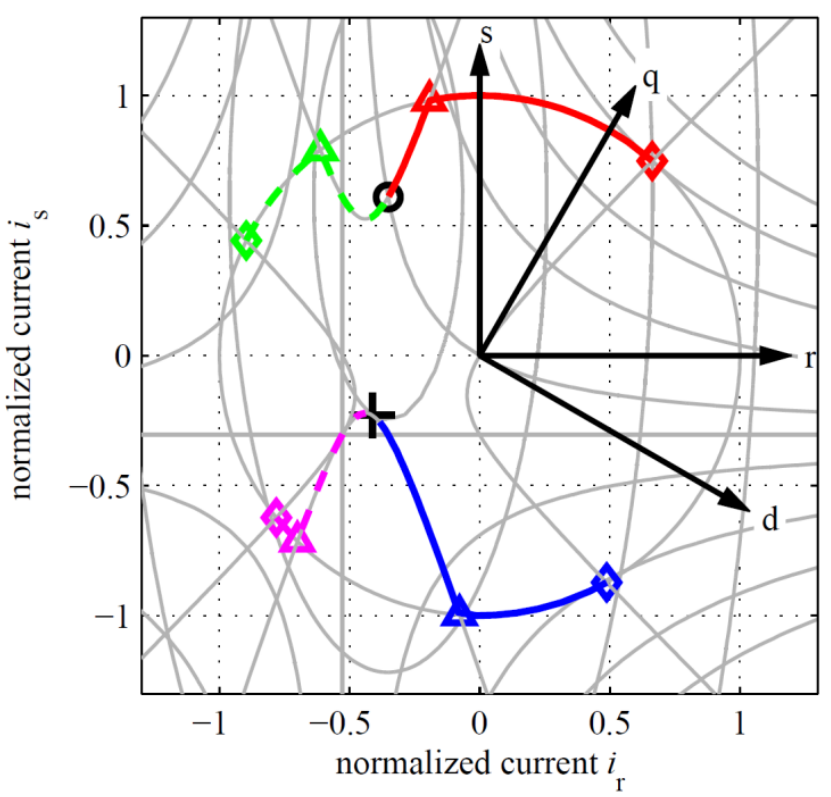

Fig. 4. Maximum torque trajectories. Solid red: main motoring mode. Solid blue: main generating mode. Dashed: secondary maximum torque trajectories. Diamonds: MTPA points resp. start of mode 2 operation. Triangles: start of mode 3 operation. Circle: point $\left(i_{\mathrm{r} \infty}, i_{\mathrm{s} \infty}\right)$. Plus sign: point $\left(i_{\mathrm{r}+}, i_{\mathrm{s}+}\right)$.

trajectory leaves the current circle again without including the point $\left(i_{\mathrm{r} \infty}, i_{\mathrm{s} \infty}\right)$ or the point $\left(i_{\mathrm{r}+}, i_{\mathrm{s}+}\right)$ in that segment, both signifying the end of the maximum torque trajectory. However, the introduced example machine behaves "ordinary". An example of a machine exhibiting the described mode order is $\psi_{\mathrm{PM}}=0.675, \zeta=2, \beta=-25^{\circ}$.

\section{G. Discussion}

Fig. 4 shows the maximum torque trajectories of the example machine. The solid lines indicate the maximum torque trajectories starting from the primary MTPA points, where red is the main motoring mode and blue is the main generating mode. The dashed lines indicate the maximum torque trajectories starting from the secondary MTPA points. The same convention is used in Fig. 5 which shows the normalized torque $t$ and the normalized power $p$ over normalized speed.

Based on these two figures, some further statements can be made. As can be seen in Fig. 5, the maximum torque trajectories starting at secondary MTPA points (dashed lines) exhibit worse performance at low speed than those starting at the primary MTPA points. However, at $\omega \approx 1.8$, best generating performance is delivered by the maximum trajectory that started at a secondary MTPA point (green dashed line).

In generating mode, trajectories should be switched at $\omega \approx 1.8$ to ensure maximum absolute torque. The blue solid line must be left at the latest when reaching $\omega \approx 3.5$. Then, the point $\left(i_{\mathrm{r}+}, i_{\mathrm{s}+}\right)$ is reached, where the trajectory ends.

\section{DESIGN Plane}

Similar to [2], design planes can be introduced for PMSM with arbitrary displacement angle $\beta$. Due to the fact that there are three independent parameters $\psi_{\mathrm{PM}}, \zeta$ and $\beta$, one of it has to be fixed in order to draw a plane.
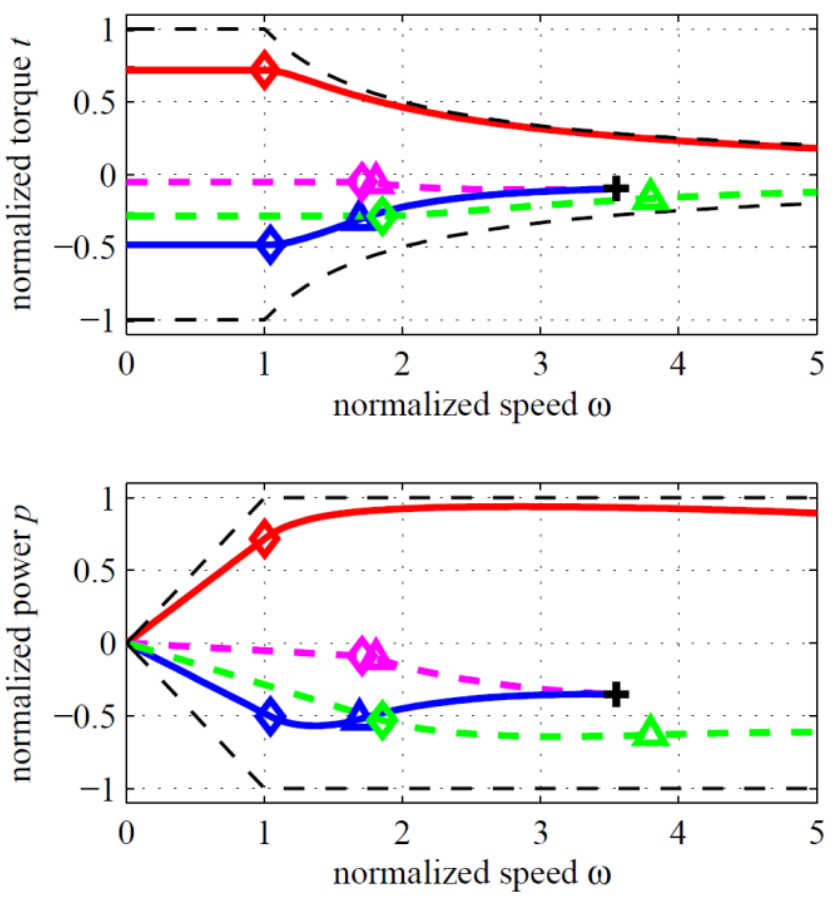

Fig. 5. Normalized torque $t$ and normalized power $p$ over normalized speed. Solid red: main motoring mode. Solid blue: main generating mode. Dashed: performance of secondary trajectories. Diamonds: start of mode 2 operation. Triangles: start of mode 3 operation. Plus sign: maximum speed of secondary trajectories.

The following two Sections describe a possible design strategy for two-part rotor motors by iteratively analyzing the two parameter planes that are described below.

\section{A. The $\psi_{P M}-\beta-$ Plane $(\zeta=$ const $)$}

Regarding two-part rotors, it is apparent that saliency is constant, independent of the displacement of the two rotor parts. As a first step when designing a motor with displaced reluctance axis, it is obvious to fix the saliency parameter to a constant value. Figs. 6 to 8 show a number of important performance data in the $\psi_{\mathrm{PM}}-\beta$ - plane for a fixed value of $\zeta=4$, which is a realistic value for two-part rotor motors [4].

As explained in Section II $B$, base values of the normalization, such as the reference stator flux linkage, are specified by the application and hence are constant when comparing different rotors. This means, a low value of $\psi_{\mathrm{PM}}$ is equivalent to low PM induced air gap flux density and hence to low magnet cost. Furthermore, $\psi_{\mathrm{PM}}$ is proportional to open loop voltage (see (7)-(9)), so reducing $\psi_{\mathrm{PM}}$ also improves safety.

Fig. 6 shows mode 1 maximum motoring torque respectively base speed power in motoring mode. For a fixed desired torque, $\psi_{\mathrm{PM}}$ is lowest at about $\beta=60^{\circ}$. This means, the torque-to-magnet-cost-ratio is highest at that angle. Compared to conventional machines with $\beta=90^{\circ}$, $\psi_{\mathrm{PM}}$ can be reduced by about $10 \%$. Investigating this plane at other values of $\zeta$ shows similar behavior.

As can be seen in Fig. 7, generating torque at that displacement angle is distinctly lower, depending on the normalized magnet flux linkage $\psi_{\mathrm{PM}}$ only about 0.7 to 0.8 times the absolute value of motoring torque due to the nonsymmetric structure of the rotor. Obviously, this is the price that has to be paid for minimizing $\psi_{\mathrm{PM}}$. 


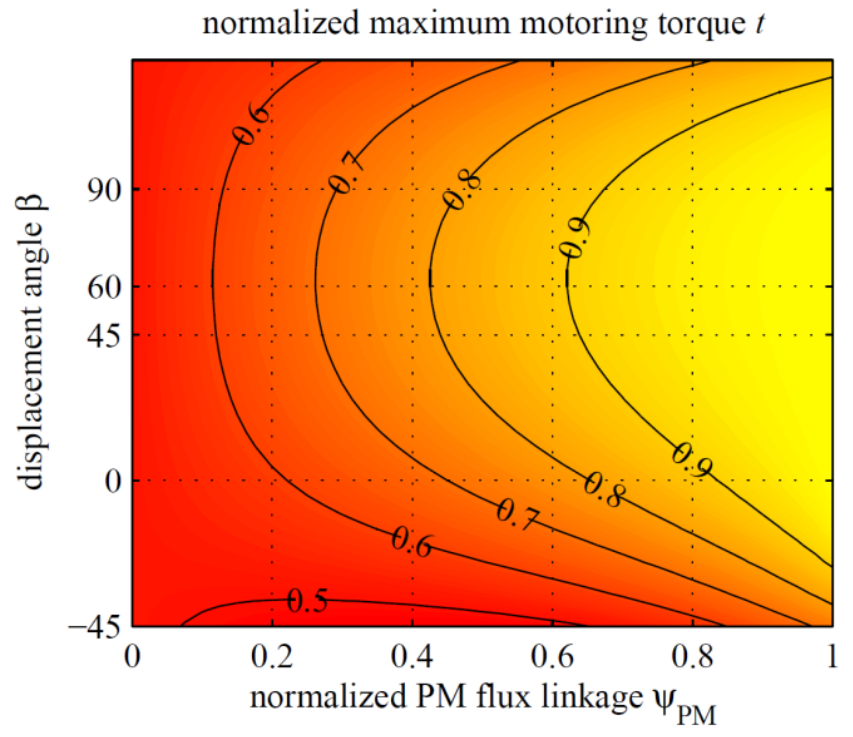

Fig. 6. Normalized maximum motoring torque $t$ for a fixed saliency value of $\zeta=4$.

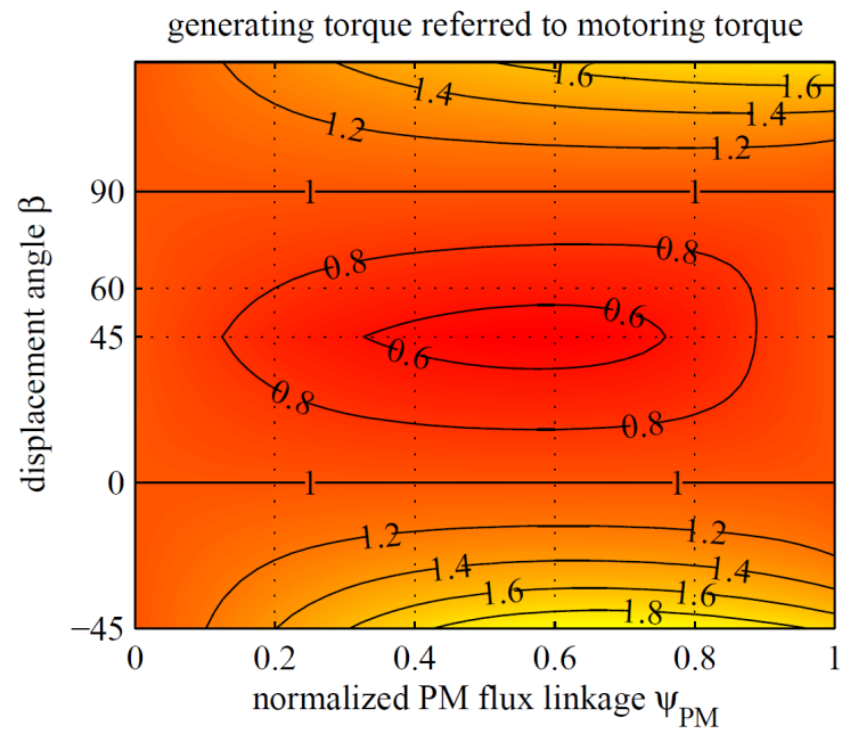

Fig. 7. Maximum generating torque referred to maximum motoring torque for a fixed saliency value of $\zeta=4$. See also Section II $E$.

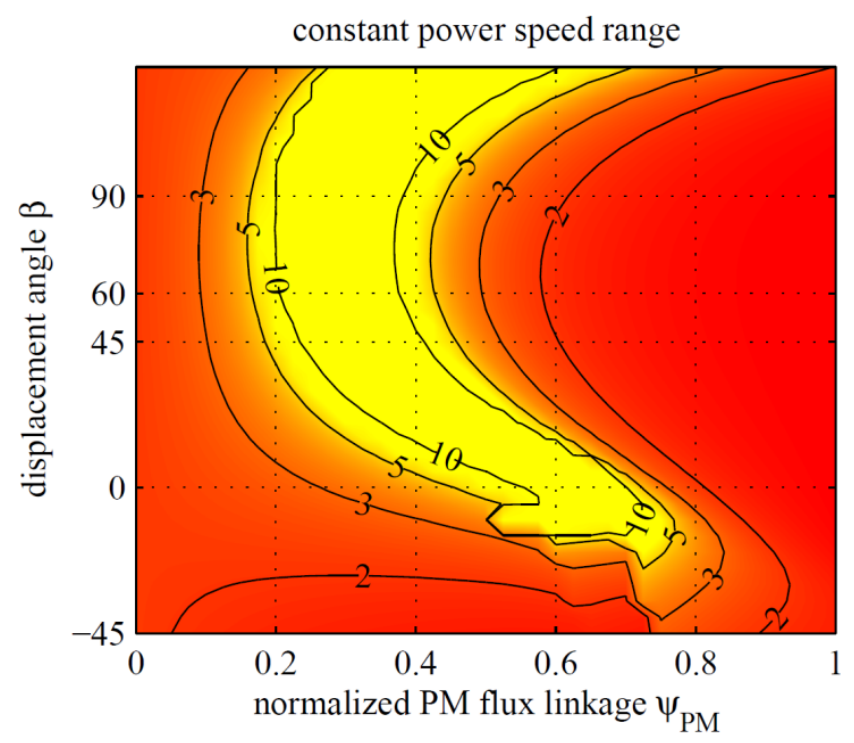

Fig. 8. Constant power speed range for a fixed saliency value of $\zeta=4$.

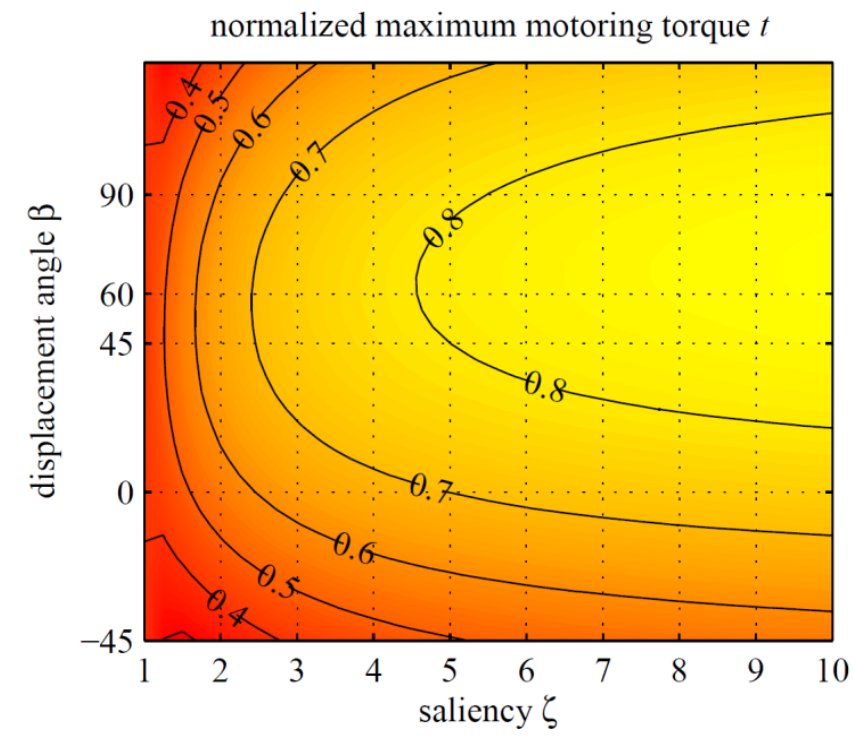

Fig. 9. Normalized maximum motoring torque $t$ for a fixed PM flux linkage value of $\psi_{\mathrm{PM}}=0.4$.

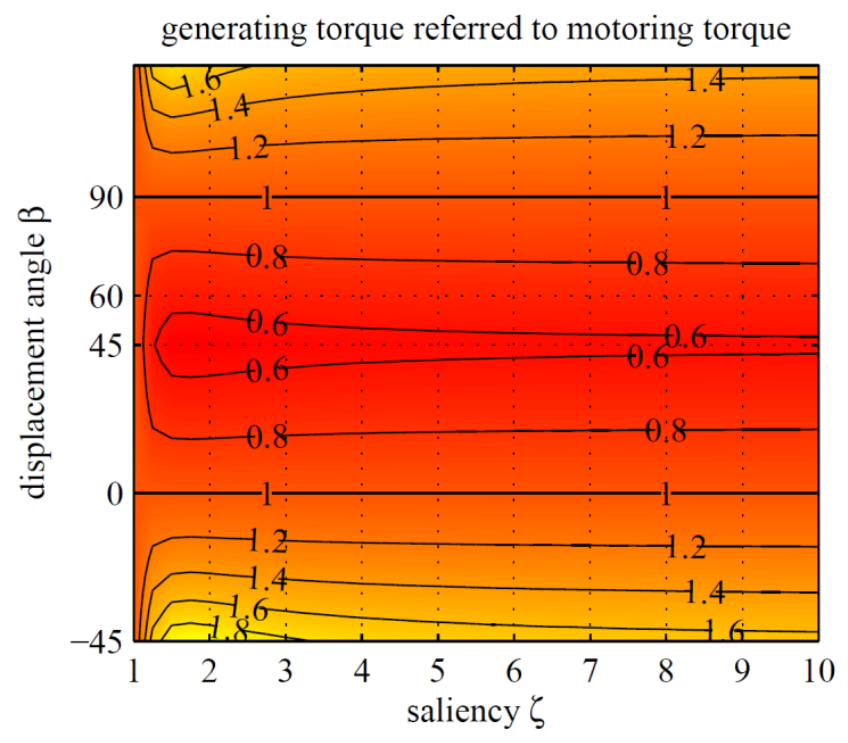

Fig. 10. Maximum generating torque referred to maximum motoring torque for a fixed PM flux linkage value of $\psi_{\mathrm{PM}}=0.4$. See also Section II $E$.

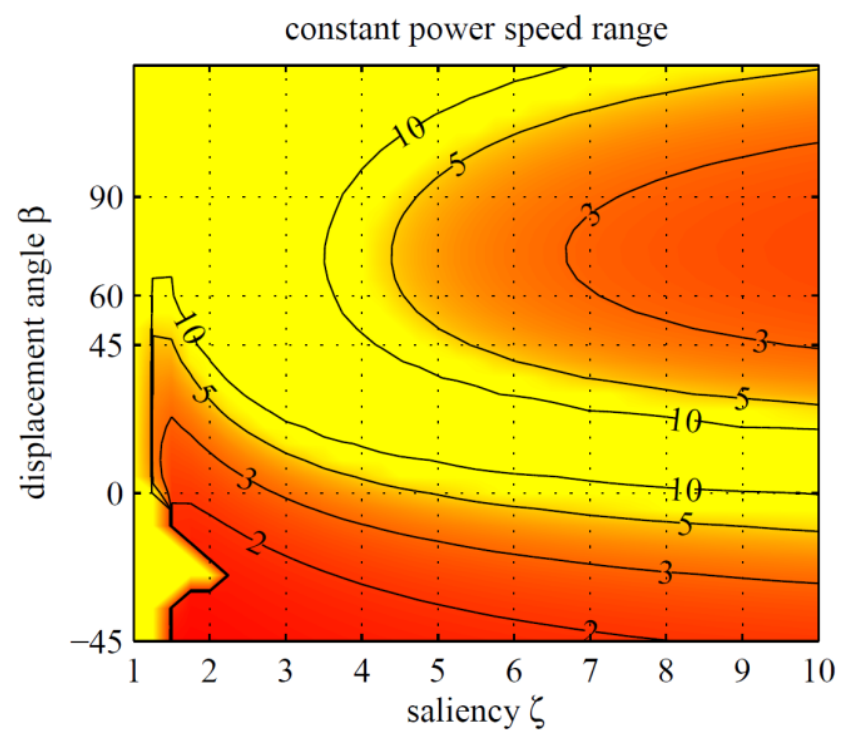

Fig. 11. Constant power speed range for a fixed PM flux linkage value of $\psi_{\mathrm{PM}}=0.4$. 
Note the lines at $\beta=0^{\circ}$ and $\beta=90^{\circ}$, indicating regular PMSM with equal motoring and generating performance. Machines with $\beta<0^{\circ}$ and $\beta>90^{\circ}$ exhibit better performance in generating mode and are therefore best used as generators. This behavior was already discussed in Section II $E$.

Fig. 8 shows the constant power speed range (CPSR, [2]) of machines with arbitrary $\beta$ and $\zeta=4$. Motors for electric traction require at least a CPSR of 3 to 4 [1], [4]. Motors with $\beta=60^{\circ}$ and $\beta=90^{\circ}$ show similar CPSR behavior.

\section{B. $\quad$ The $\zeta-\beta-$ Plane $\left(\psi_{P M}=\right.$ const $)$}

After determining a displacement angle $\beta$ and a normalized PM flux linkage $\psi_{\mathrm{PM}}$ using the $\psi_{\mathrm{PM}}-\beta-$ plane, the individual length of the two rotor parts may be changed. This causes a variation of the parameter $\zeta$. The effects are discussed using Figs. 9 to 11 . Exemplary, $\psi_{\mathrm{PM}}$ is set to 0.4 .

Fig. 9. shows mode 1 operation torque respectively base speed power in motoring mode. Again, for a fixed desired torque, $\psi_{\mathrm{PM}}$ is lowest at about $\beta=60^{\circ}$.

From Fig. 10. can be concluded that the maximum torque ratio of motoring to generating mode is rather a function of $\beta$ than of $\zeta$ as the contour lines are quite constant for $\zeta>1.5$.

The CPSR in the $\zeta-\beta$ - plane with $\psi_{\mathrm{PM}}=0.4$ is given in Fig. 11. Again, motors with $\beta=60^{\circ}$ and $\beta=90^{\circ}$ show similar CPSR behavior.

\section{CONCLUSION}

This paper accomplished two goals. First, it set up a unified theory to describe any synchronous machine, whose reluctance axis is displaced to the rotor excitation axis by any arbitrary but constant angle. Optimal torque control strategies have been derived from that set of equations and some special characteristics of those machines which are unknown from conventional PMSM have been demonstrated.

Second, from a set of possible motor configurations, different design planes have been plotted and a strategy to design motors has been developed. It was shown that machines with displacement angles of about $60^{\circ}$ need up to $10 \%$ less permanent magnet material than conventional machines, yielding comparable motor performance. Although generating performance deteriorates by about $20 \%$ to $30 \%$, those machines could be a promising alternative in applications which mostly require motoring mode, such as traction drives.

\section{REFERENCES}

[1] D. F. Gosden, B. J. Chalmers, and L. Musaba, "Drive system design for an electric vehicle based on alternative motor types," 5th Int Conf. on Power Electronics and Variable-Speed Drives, 1994, pp. $710-715$

[2] W. L. Soong and T. J. E. Miller, "Theoretical limitations to the fieldweakening performance of the five classes of brushless synchronous AC motor drive," 6th Int. Conf. on Electrical Machines and Drives, 1993, pp. 127-132

[3] N. Bianchi and S. Bolognani, "Performance analysis of an IPM motor with segmented rotor for flux-weakening applications," 9th Int. Conf. on Electrical Machines and Drives, 1999, pp. 49-53

[4] B. J. Chalmers, L. Musaba, and D. F. Gosden, "Variable-frequency synchronous motor drives for electric vehicles," IEEE Trans Industry Applications, vol. 32, no. 4, pp. 896-903, Jul/Aug 1996

[5] D. F. Gosden, B. J. Chalmers, and L. Musaba, "Field weakening performance of a synchronous motor with two-part rotor," 8th Int. Conf. on Electrical Machines and Drives, 1997 pp. 244-247

[6] B. J. Chalmers, R. Akmese, and L. Musaba, "Design and fieldweakening performance of permanent-magnet/reluctance motor with two-part rotor," IEE Proc. - Electric Power Applications, vol. 145, no. 2, pp. 133-139, Mar 1998

[7] N. Bianchi, S. Bolognani, and B. J. Chalmers, "Salient-rotor PM synchronous motors for an extended flux-weakening operation range," IEEE Trans. Industry Applications, vol. 36, no. 4, pp. 1118 1125, Jul/Aug 2000

[8] S. A. Randi and S. Astier, "Parameters of Salient Pole Synchronous Motor Drives with Two-part Rotor to Achieve a Given Constant Power Speed Range," 32nd Annual Power Electronics Specialists Conf., 2001, pp. 1673-1678

[9] X. Chen, "Analysis on Different Rotor Structure Synchronous Motor Vector Control," 2nd Int. Conf. on Intelligent Computation Technology and Automation, 2009, pp. 14-17

[10] E. K. Beser, S. Camur, B. Arifoglu, and E. Beser, "Analysis and Application of a Hybrid Motor Structure Convenient to Modify the Magnet and Reluctance Torques on the Rotor," in J. Elec. Eng. \& Tech., vol. 7, no. 3, pp. 349-357, 2012

[11] W. L. Soong, " Design and Modelling of Axially-Laminated Interior Permanent Magnet Motor Drives for Field-Weakening Applications," Ph.D. dissertation, Dept. Electron. Electr. Eng., Univ. Glasgow, UK, 1993.

[12] D. Schröder, Elektrische Antriebe - Regelung von Antriebssystemen, Berlin, Heidelberg, Germany, SpringerLink, 2009

\section{BIOGRAPHIES}

Patrick Winzer was born in Lörrach, Germany in 1985. He graduated from Karlsruhe Institute of Technology (KIT) in 2011 and works since then at the Institute of Electrical Engineering at KIT as Ph.D. student in the field of electric traction drives.

Martin Doppelbauer studied electrical engineering at the University of Dortmund in Germany and graduated 1995 with a doctorate thesis on the analytical calculation of universal motors. He worked in the industry from 1995 until 2010. Since 2011 he is professor at the Institute of Electrical Engineering (ETI) of the Karlsruhe Institute of Technology (KIT) where he holds a chair for Hybrid Electric Vehicles. Since 1996 Martin Doppelbauer also works in international standardization of industrial motors and holds several positions as chairman, convenor and speaker in DKE, CENELEC and IEC. He is actively involved in the Association for Electrical, Electronic \& Information Technologies (VDE) and chairman of the "Fachbereich A1 - Electrical Machines and Drives“. 